BABELAO 3 (2015), p. 33-43

(C) ABELAO (Belgium)

\title{
«Car tout [homme] au feu sera salé ». Un réexamen textuel de Marc 9,49
}

Par

\section{Audrey Wauters}

Université Catholique de Louvain, Louvain-la-Neuve

L a publication de la vingt-huitième édition du Nestle-Aland témoigne du renouvellement continu de la recherche en critique textuelle du Nouveau Testament ${ }^{1}$. Mais malgré les nouveautés apportées ${ }^{2}$, d'aucuns regretteront sans doute que certains aspects de ce domaine de recherche demeurent quasiment

1 Pour un récent état de la question, voir B.D. EHRMAN - M.W. Holmes (eds), The Text of the New Testament in Contemporary Research. Essays on the Status Quaestionis, Leiden, 2013, $2^{\text {nd }}$ ed.

2 Ces nouveautés ne concernent cependant pas le propos de cet article, puisqu'elles portent uniquement sur les Catholiques : «the 28th edition of the Nestle-Aland had to accomplish two different tasks. First, the apparatus had to be revised thoroughly to give it more clarity and make it easier to use. Secondly, the text-critical insights and decisions resulting from work on the Editio Critica Maior of the Greek New Testament had to be incorporated. As a consequence of these alterations, which so far concern only the Catholic Letters, 
inchangés depuis plus d'un siècle. Force est en effet de constater que la primauté du texte alexandrin continue à se maintenir depuis son appréciation par Westcott et Hort à la fin du XIX ${ }^{\mathrm{e}}$ siècle ${ }^{3}$, et ce d'autant plus lorsqu'une leçon est attestée par le fameux Codex Vaticanus. Par conséquent, puisqu'ils avaient jugé

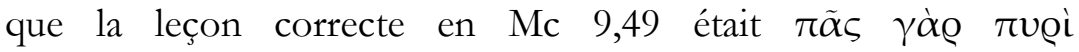

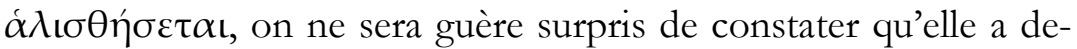
puis été reprise par les principales éditions critiques du texte néotestamentaire ${ }^{4}$.

Cette curieuse expression, unique dans tout le Nouveau Testament, a suscité nombre de commentaires ${ }^{5}$, tant exégétiques que théologiques. Situé entre un enseignement à propos d'«entrer dans la vie » et une parole sur le sel, ce logion est sans conteste " one of the most perplexing pronouncements in the Synoptic tradition $^{6}$ ", et "faisait déjà problème aux premiers siècles du christianisme ${ }^{7} »$. Sa difficulté est d'ailleurs telle qu'il arrive qu'elle se ressente jusqu'en traduction ${ }^{8}$. Pourtant, il nous semble que cette difficulté réside essentiellement dans le choix de cette variante pour un lieu variant qui n'en compte pas moins d'une ving-

the Nestle-Aland has for the first time in its history a different presentation for different parts of the text», cf. B. and K. AlAND - J. KARAVIDOPOUlos C.M. Martini - B.M. MetzGer (eds), Novum Testamentum Graece, Stuttgart, 2012, $28^{\text {th }}$ ed. revised, p. 48*.

${ }^{3}$ Jusqu'alors, c'était évidemment le texte byzantin - le texte majoritaire qui faisait autorité, ainsi qu'en témoigne encore W. BOUSSET, Textkritische Studien zum Neuen Testament (Texte und Untersuchungen zur Geschichte der altchristlichen Literature 11/4), Leipzig, 1894, p. 98 pour Mc 9,49 plus d'une dizaine d'années après la publication de The New Testament in the Original Greek.

${ }^{4}$ On retrouve ainsi cette leçon dans l'édition de Legg, GNT ${ }^{4}$ (qui indique avoir choisi la leçon avec $\{\mathrm{B}\}$, c'est-à-dire avec une relativement grande certitude) et $\mathrm{NA}^{28}$, tout comme chez une écrasante majorité d'exégètes modernes.

${ }^{5}$ Ces commentaires se sont surtout développés à partir du Moyen Âge, ainsi que le note D. FraYer-GrIGGS, "Everyone Will Be Baptized in Fire": Mark 9.49, Q 3.16, and the Baptism of the Coming One ", Journal for the Study of the Historical Jesus 7 (2009), p. 254-285 (260-261) : « unlike Matthew, Luke, and the early Church Fathers, who were perhaps allergic to this saying's ambiguity and were thus inclined to evade the enigmatic expression, exegetes from the medieval period to the present offer up a cacophony of dissonant voices, which only succeed in drowning out one another $»$.

${ }^{6}$ IBID., p. 254.

7 J. DuponcheELE, "“Car chacun sera salé au feu...” (Mc 9,49-50) », Revue théologique de Louvain 39 (2008), p. 67-79 (68).

${ }^{8}$ La grande majorité des traductions sont sensiblement similaires (« car tous seront salés par le feu » pour la $B J$, «car tout homme sera salé de feu » pour la Bible Segond, ou "car chacun sera salé au feu » pour la TOB). Quelques auteurs ont cependant pris certaines libertés lorsqu'ils ont eu à traduire cette expression, par exemple J. CAZEAux, Marc. Le lion du désert. Essai (Lectio Divina 252) Paris, 2012, p. 172 : « oui, chacun par le feu aura (la saveur du) sel ». 
taine ${ }^{9}$, et ce d'autant plus parce que la péricope (v. 41-50) se révèle au final n'être qu'une collection relativement hétéroclite de logia ${ }^{10}$.

C'est pourquoi cet article propose un réexamen de l'ensemble des variantes en Mc 9,49, à la lumière des trois étapes de la critique textuelle (critique verbale, critique externe et critique interne), afin d'en déterminer la leçon la plus ancienne. Notre intérêt portera tout particulièrement sur les résultats de la critique interne, parce qu'elle seule permet de poser un jugement de valeur sur les variantes. En cela, nous nous détacherons de la grande majorité des textualistes qui, comme nous le verrons cidessous, s'en tiennent globalement à ce que la critique verbale peut révéler de ce verset.

\section{Les variantes}

L'établissement des variantes permet dans un même temps de déterminer les plus importantes (certaines des variations mineures seront expliquées par la suite) et de repérer les différents témoins et versions qui les présentent.

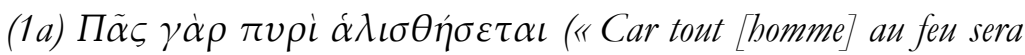
salé»)

\begin{tabular}{l|l} 
Onciaux & B.03 L.019 $\Delta .0370274$ \\
\hline Minuscules & $\begin{array}{l}\mathrm{f}^{1.13} 2228^{*} 6173205206229^{*} 251^{*} 258 \\
43548556569770010061278^{*}\end{array}$ \\
\hline Lectionnaire & 1260 \\
\hline Versions & syr $^{\mathrm{s}}$ sa bo ${ }^{\mathrm{pt}}$ arm geo \\
\hline Pères de l'Église & Théodore d'Héraclée Didyme
\end{tabular}

\begin{tabular}{|c|c|}
\hline S.01 1342 & 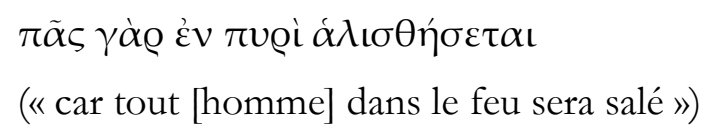 \\
\hline W.032 & 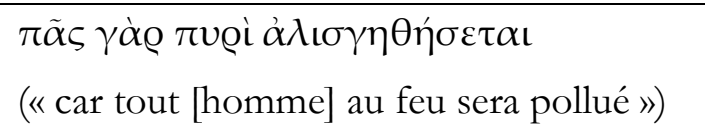 \\
\hline
\end{tabular}

9 À ce propos, on s'étonnera grandement de ne pas retrouver ce lieu variant dans H. GREEVEN - E. GüTING, Textkritik des Markusevangeliums (Theologie. Forschung und Wissenschaft 11), Münster, 2005.

10 C. Focant, L'évangile selon Marc (Commentaire biblique. Nouveau Testament 2), Paris, 2004, p. 364 : «la série de consignes rassemblées dans ces versets est passablement disparate et le lien entre elles est lâche. L'unification est formelle et principalement réalisée par des mots-crochets ou des tournures de phrase semblables ». C'est également l'opinion de P. BENOIT et M.É. BOISMARD, Synopse des quatre évangiles en français, vol. 2, Paris, 1972, p. 266 ; M.E. Boring, Mark. A Commentary (The New Testament Library), LouisvilleLondon, 2006, p. 279 et S. LÉGASSE, L'évangile de Marc (Lectio Divina. Commentaires 5), vol. 2, Paris, 1997, p. 578. 


\begin{tabular}{|c|c|}
\hline 1188125220230 & 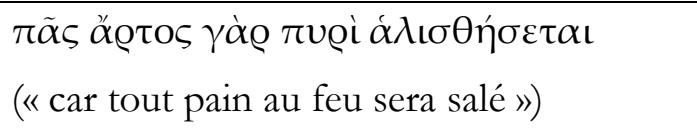 \\
\hline 4652 & 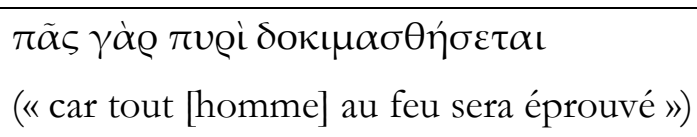 \\
\hline $\mathrm{vg}^{\mathrm{ms}} \mathrm{bo}^{\text {aliq }}$ & 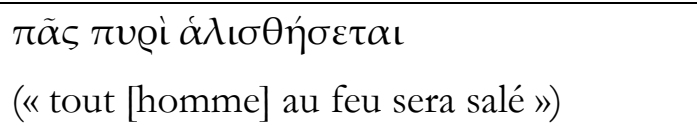 \\
\hline$g^{1}$ & examinantur (« ils sont pesés ») \\
\hline
\end{tabular}

TO

TCés W.032 $\mathrm{f}^{1.13} 28 * 565700 \mathrm{arm}$ geo

TA S.01 B.03 L.019 $\Delta .037 \mathrm{sa}$

TB

Autres syr $^{\mathrm{s}}$ bo $^{\mathrm{pt}}$ Théodore d'Héraclée Didyme

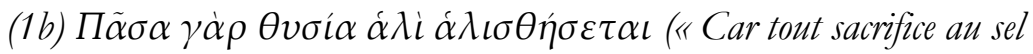
sera salé»)

\begin{tabular}{l|l} 
Oncial & D.05 \\
\hline Pères de l'Église & Ambroise Chromace \\
$a$ & $\begin{array}{l}\text { omnis hostia insalabitur } \\
\text { (« toute victime sera salée ») }\end{array}$ \\
\hline aurc & $\begin{array}{l}\text { omnis enim uictima salietur } \\
\text { (« en effet, toute victime sera salée ») }\end{array}$ \\
\hline$b f f^{2} i$ & $\begin{array}{l}\text { omnis enim uictima sale salietur } \\
\text { (« en effet, toute victime sera salée par le } \\
\text { sel ») }\end{array}$ \\
\hline$d$ & $\begin{array}{l}\text { omne enim sacrificium sali salietur } \\
\text { (« en effet, tout sacrifice au sel sera salé ») }\end{array}$ \\
\hline$k$ & $\begin{array}{l}\text { omnia [autem] substantia consumitur } \\
\text { (« [or,] tout être est consumé ») }\end{array}$
\end{tabular}

TO D.05a aurbcdff $i k$

TCés

TA

TB

Autres Ambroise Chromace 


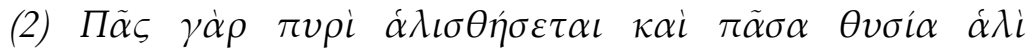
$\alpha \lambda \iota \sigma \theta \dot{\eta} \sigma \varepsilon \tau \alpha \iota$ ("Car tout [homme] au feu sera salé et tout sacrifice an sel sera salé »)

\begin{tabular}{|c|c|}
\hline Onciaux & $\begin{array}{l}\text { А.02 E.07 F.09 G.011 H.013 K.017 N.022 } \\
\text { Г.036 П.041 } \Sigma .042 \text { Ф.043 }\end{array}$ \\
\hline Minuscules & $\begin{array}{l}1328^{\mathrm{c}} 157180543597828892 \quad 1010^{*} 1071 \\
124112431278^{\mathrm{mg}} 1292142415052542\end{array}$ \\
\hline Texte reçu & Byz. \\
\hline Lectionnaires & Lect \\
\hline Versions & $l \mathrm{vg}^{\mathrm{cl}} \mathrm{syr}^{\mathrm{ph}} \mathrm{bo}^{\mathrm{pt}}$ eth slav \\
\hline
\end{tabular}

\begin{tabular}{|c|c|}
\hline C.04 & 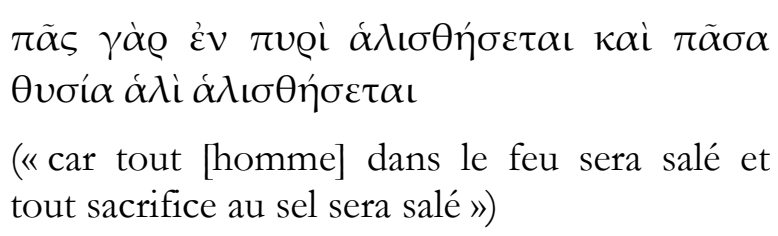 \\
\hline$\Theta .038$ & 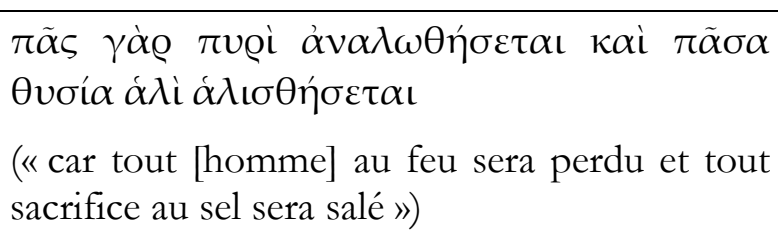 \\
\hline$\Psi .044$ & 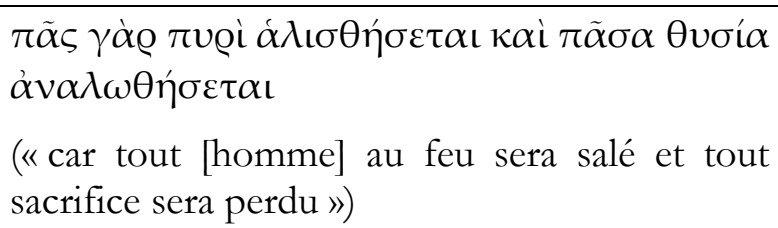 \\
\hline X.033 & 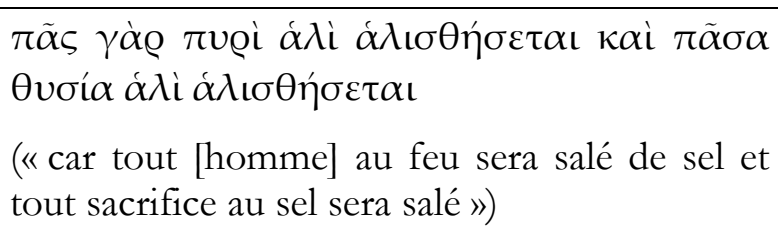 \\
\hline 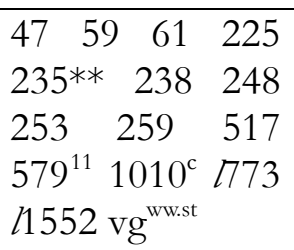 & 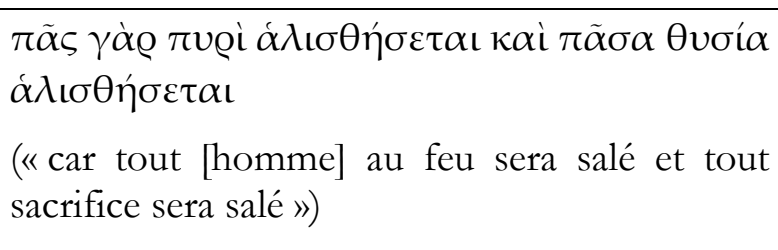 \\
\hline 1673 & 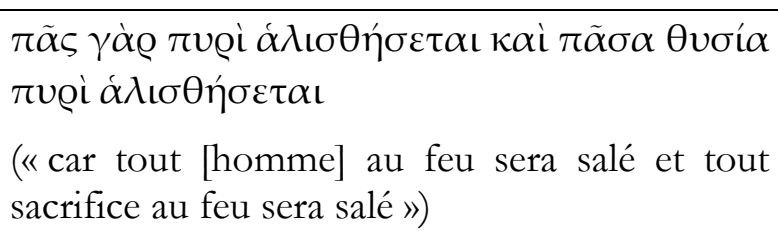 \\
\hline
\end{tabular}

11 Nous remercions J. K. Elliott de nous avoir confirmé que le Ms. 579 donne bien la leçon longue, ainsi qu'indiqué dans l'édition de Legg et NA28. Par conséquent, $\mathrm{GNT}^{4}$ se trompe en lui attribuant la leçon brève $\pi \tilde{\alpha} \varsigma \gamma \dot{\alpha} \varrho$ Tu@i $\dot{\alpha} \lambda \iota \sigma \theta \dot{\gamma} \sigma \varepsilon \tau \alpha$. 


\begin{tabular}{|c|c|c|}
\hline$f$ & & $\begin{array}{l}\text { omnis enim igne salietur et omne sacrificium } \\
\text { sale salietur } \\
\text { (« en effet, tout [homme] sera salé par le feu et } \\
\text { tout sacrifice sera salé par le sel») }\end{array}$ \\
\hline$q$ & & $\begin{array}{l}\text { omnis enim igne salietur et omnis uictima sali } \\
\text { salietur } \\
\text { (« en effet, tout [homme] sera salé par le feu et } \\
\text { toute victime au sel sera salée ») }\end{array}$ \\
\hline$r^{2}$ & & $\begin{array}{l}\text { omnis enim igne salietur et omnis uctima salie- } \\
\text { tur } \\
\text { (« en effet, tout [homme] sera salé par le feu et } \\
\text { toute victime sera salée ») }\end{array}$ \\
\hline & TO & $r^{2}$ \\
\hline & TCés ${ }^{12}$ & $\Theta .03828^{\epsilon}$ \\
\hline & TA & 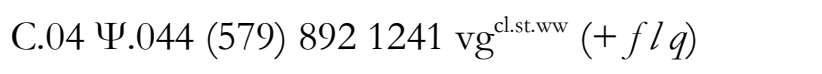 \\
\hline & TB & A.02 Byz. \\
\hline & Autres & syr $^{\mathrm{p} . \mathrm{h}} \mathrm{bo}^{\mathrm{pt}}$ eth \\
\hline
\end{tabular}

Ce «tour d'horizon» de l'ensemble des variantes qui apparaissent en Mc 9,49 a permis de montrer que seules trois variantes se dégagent vraiment ${ }^{13}$ : deux variantes brèves $(\pi \tilde{\alpha} \varsigma$ $\gamma \dot{\alpha} \varrho$

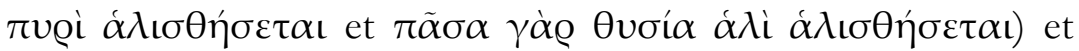

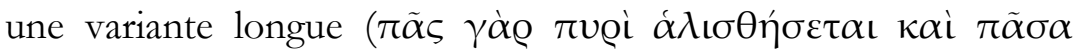
$\theta v \sigma i ́ \alpha \alpha \dot{\alpha} \lambda \dot{\alpha} \lambda \iota \sigma \theta \eta \dot{\sigma \varepsilon \tau} \alpha \iota)$.

\section{Critique verbale}

Lorsque se pose la question de savoir laquelle de ces trois variantes est la plus ancienne, il apparait que bon nombre de textualistes se sont contentés de la seule critique verbale. Ainsi l'explication de Metzger ${ }^{14}:$ « at a very early period a scribe, having

${ }^{12}$ En barrant ces témoins, nous indiquons que le texte césaréen n'a ici aucune variante, qu'il n'est pas attesté : les témoins $\Theta .03828 \mathrm{f}^{1} \mathrm{f}^{13}$ etc. transmettent le texte césaréen quand leur texte diffère de celui des autres types, en particulier du type byzantin.

${ }^{13}$ FrAYER-Griggs, p. 257. La leçon de $k$, « omnia [autem] substantia consumitur », n'est pas indépendante comme le suggère GNT $^{4}$ qui la fait précéder du symbole //, mais est la conséquence d'une confusion de lettres : le copiste a

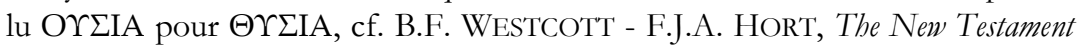
in the Original Greek, vol. 2 : Introduction and Appendix, New York, 1882 (repr. Graz, 1974), \142.

14 B.M. Metzger, A Textual Commentary on the Greek New Testament. A Companion Volume to the United Bible Societies' Greek New Testament, Stuttgart, 1989, $3^{\text {rd }}$ ed., p. 103. Son explication sera reprise telle quelle par FOCANT, p. 363. 
found in $\mathrm{Lv} 2: 13$ a clue to the meaning of Jesus' enigmatic state-

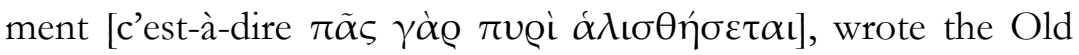
Testament passage in the margin of his copy of Mark. In subsequent copyings the marginal gloss was either substituted for the words of the text, thus creating reading (2) [c'est-à-dire $\pi \tilde{\alpha} \sigma \alpha$

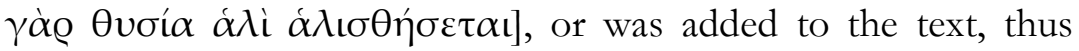

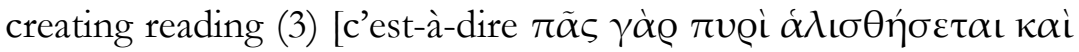

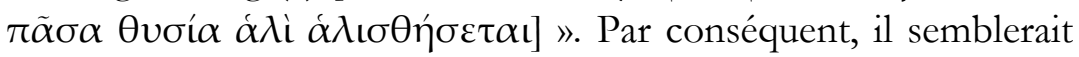
que tout puisse se résumer à la simple addition d'une glose explicative au texte évangélique.

Si cette explication se tient plutôt bien pour la leçon longue, une autre peut cependant être avancée concernant la leçon brève

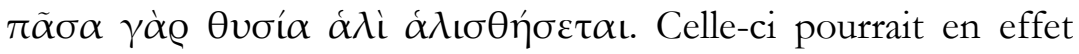
tout aussi bien s'expliquer par une correction harmonisante sur la

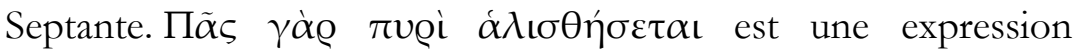
unique, qui ne se retrouve pas dans les parallèles synoptiques ${ }^{15}$ (la mise en garde en Mt 5,13 et Lc 14,34-35 et la parole sur le sel en Mt 18,6-11 et Lc 17,1-2) ; un copiste aurait pu remplacer ce verset par le texte vétérotestamentaire - quoique quelque peu modifié -, croyant avoir sous les yeux une copie corrompue. Une omission par homoiotéleuton sur AIПA entre $\sigma \beta \varepsilon ́ v v v \tau \alpha \iota \pi \tilde{\alpha} \varsigma$ et $\kappa \alpha i$

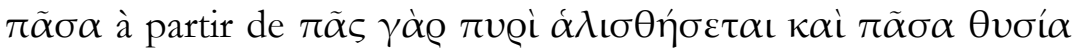

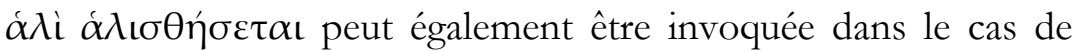
cette leçon.

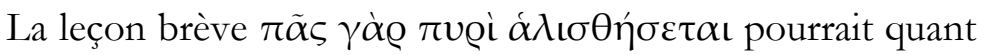
à elle s'expliquer par un passage du même au même sur le verbe $\alpha \lambda_{\iota \sigma} \theta \dot{\eta} \sigma \varepsilon \tau \alpha \iota^{16}$, à partir de la leçon longue : après avoir copié la première moitié du verset, l'œil du copiste aurait glissé sur la deuxième occurrence de ce verbe. Néanmoins, il est certain qu'il est plus vraisemblable que la leçon longue résulte de la combinaison

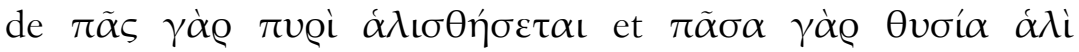
$\alpha \lambda \iota \sigma \theta \eta \dot{\sigma \varepsilon \tau} \alpha$ ı, d'autant plus que le phénomène de parablepse n'explique pas l'apparition de l'autre leçon brève.

Par ailleurs, on remarquera le grand nombre de variations qui apparaissent au sein même des différentes leçons. Dans le cas

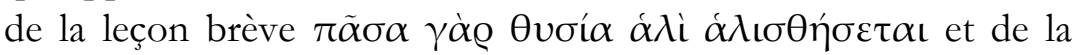
leçon longue, la plupart de celles-ci peuvent être considérées comme sans réel intérêt : à l'exception de $\Theta .038$ et de $\Psi .044^{17}$ qui

${ }^{15}$ FraYeR-Griggs, p. 255: «Mark 9.49 is [...] one of the few Markan texts that neither Matthew nor Luke incorporated into his Gospel ».

${ }^{16}$ H. ZimmermanN, «Mit Feuer gesalzen werden: Eine Studie zu Mk 9,49 ", Theologische Quartalschrift 139 (1959), p. 28-39 (30-31): « vorab liegt bei der von $\mathrm{S}$ B u. a. bezeugten, kürzeren Textform die Annahme eines alten Homoioteleutonfehlers sehr nahe, wobei das Auge des Schreibers von dem ersten

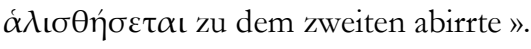

17 Pour $\Psi .044$, Metzger, p. 103 explique le passage de $\alpha \lambda \iota \sigma \theta \eta ́ \sigma \varepsilon \tau \alpha \iota$ à $\dot{\alpha} v \alpha \lambda \omega \theta \eta \dot{\sigma \varepsilon \tau \alpha \iota ~ c o m m e ~ u n e ~ c o n f u s i o n ~ d e ~ l e t t r e s ~ s u r ~ A ~} \Lambda$ IA $\Lambda$ IC, ce qui permet 
remplacent le verbe $\alpha \lambda i \zeta \zeta \omega$ par $\alpha \dot{v} \alpha \lambda i ́ \sigma \kappa \omega$, il s'agit essentiellement de l'absence ou de la présence de la conjonction $\gamma \dot{\alpha} \varrho$ ou du substantif $\alpha \alpha \varsigma^{18}$; on les considérera par conséquent comme des accidents de copie. Or, dans le cas de la leçon brève $\pi \tilde{\alpha} \varsigma$ $\gamma \dot{\alpha} \varrho$ $\pi v \varrho i ̀ ~ \alpha \Lambda \iota \sigma \theta \eta ́ \sigma \varepsilon \tau \alpha$, on constate un changement de verbe à deux

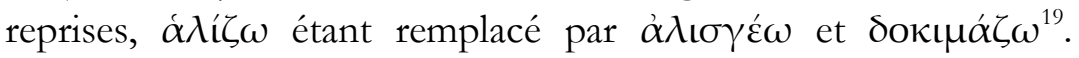
$A \lambda \iota \sigma \gamma \varepsilon \dot{\varepsilon} \omega$ présentant une forme relativement similaire à celle de $\alpha \lambda \lambda i \zeta \omega$ à l'indicatif futur passif, il peut être envisagé comme une modification involontaire. Pour ce qui est de $\delta о к \iota \mu \alpha ́ \zeta \omega$, il apparaît que cette modification résulte d'une reprise du commentaire de Théophylacte ${ }^{20}$, qui explique $\alpha \lambda \iota \sigma \theta \eta ́ \sigma \varepsilon \tau \alpha \iota$ comme signifiant

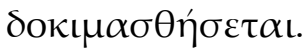

Si la critique textuelle, telle qu'appliquée par Metzger, apporte un résultat fort satisfaisant, force est de constater que d'autres solutions sont envisageables; notre examen des diverses leçons en Mc 9,49 doit donc impérativement se poursuivre. On peut toutefois exclure dès à présent la leçon longue. En effet, il apparaît assez clairement qu'elle ne peut être que la combinaison des leçons brèves, ce qui est en outre une position assez ancienne dans l'histoire de la critique textuelle ${ }^{21}$. On notera aussi que les

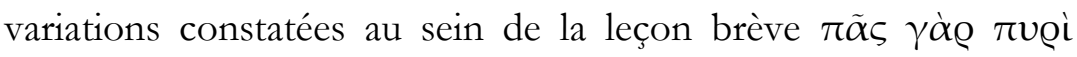
$\alpha \lambda \iota \sigma \theta \eta \dot{\sigma \varepsilon \tau} \alpha \iota$ peuvent être considérées comme un indice suggérant qu'elle a été retravaillée, et ce même s'il est possible d'y apporter une explication.

\section{Critique externe}

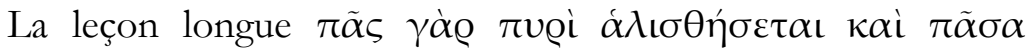

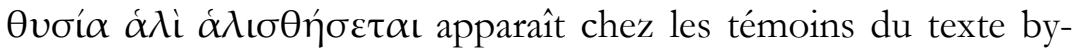
zantin dont l'Alexandrinus (A.02), ainsi que dans une partie des

du reste de comprendre l'omission de $\alpha \lambda i$. Voir aussi ZIMMERMANN, p. 30, qui donne la même explication pour $\Theta .038$. Dans ce cas, on doit supposer une leçon identique à celle de X.033 à l'origine. Par ailleurs, F.C. BURKITT, « W and $\Theta$. Studies in the Western Text of St Mark», Journal of Theological Studies 17

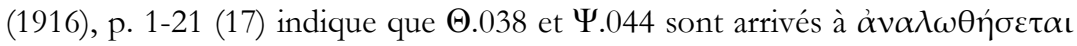
indépendamment l'un de l'autre.

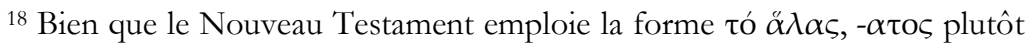
que la forme classique ó $\alpha \alpha \Lambda, \alpha \alpha \lambda$ ós, c'est cette dernière que l'on retrouve dans ces deux leçons.

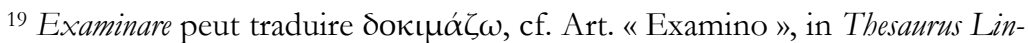
guae Latinae, vol. 5/2/2, Leipzig, 1931-1953, col. 1167. Par conséquent, nous soutenons pour le premier terme la même explication que pour le second, en supposant que le traducteur latin de $g^{1}$ a travaillé sur un manuscrit grec ainsi corrompu.

${ }^{20}$ E.W. SAunders, «Studies in Doctrinal Influences on the Byzantine Text of the Gospels », Journal of Biblical Literature 71 (1952), p. 85-92 (89).

${ }^{21}$ Ainsi WestcotT - HorT, \ 142: «the Byzantine reading can in principle be explained as a conflation of the Western and Alexandrian reading ». 


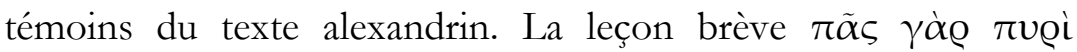
$\alpha \lambda \iota \sigma \theta \eta \dot{\sigma \varepsilon \tau} \alpha \iota$ est attestée par les témoins du texte césaréen et par une autre partie des témoins du texte alexandrin dont le Vaticanus

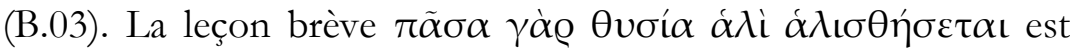
quant à elle propre aux témoins du texte occidental (grecs et vieux latins); elle est la seule à n'être attestée que par un seul type de texte, dont le meilleur témoin, le Codex de Bèze (D.05), donne un texte court.

Seules les leçons brèves apparaissent chez les Pères de l'Église :

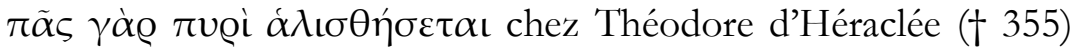

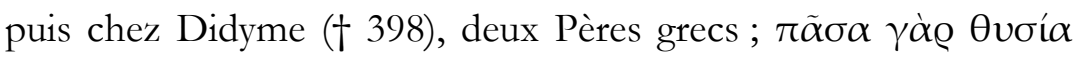
$\alpha \alpha \lambda i \alpha \Lambda ı \sigma \eta ́ \sigma \varepsilon \tau \alpha \iota$ chez Ambroise († 397) puis chez Chromace $(\dagger$ 407), deux Pères latins.

La critique externe fait ainsi ressortir que les leçons brèves étaient vraisemblablement en concurrence au $\mathrm{IV}^{\mathrm{e}}$ siècle, chacune en outre étant apparemment propre à une partie du monde chrétien. La leçon longue n'étant citée par aucun Père, cela renforce d'autant plus le fait qu'elle doit être écartée.

\section{Critique interne}

Si la critique verbale et la critique externe ont permis d'éliminer définitivement la leçon longue, il est indéniable qu'elles ne permettent pas à elles seules de déterminer laquelle des deux leçons brèves est la plus ancienne. Pourtant, la critique interne a rarement été appliquée à Mc 9,49 et, en la matière, on notera en particulier les propositions de Duponcheele et d'Elliott.

Duponcheele opte pour la leçon du Sinaiticus ( $\pi \tilde{\alpha} \varsigma \gamma \grave{\alpha} \varrho$ Ẻv

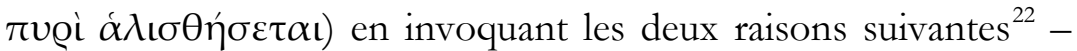
en outre corrélatives. Selon lui, S.01 résoud l'ambiguité découlant de $\pi$ voi, qui peut être envisagé aussi bien comme un datif instrumental que comme un datif locatif, grâce à la présence de la préposition $\dot{\varepsilon} v$, le sens instrumental donnant d'après lui une importance trop grande à l'action du feu au détriment de celle du $\mathrm{sel}^{23}$. Par conséquent, il invoque la règle de la lectio difficilior en faveur de S.01, parce que «il y a d'une part l'action du feu et d'autre part l'action du sel. Il faut respecter les deux, sans réduire celle du sel à celle du feu ou inversement penser que le feu aurait un pouvoir d'assaisonnement pour épicer ou conserver ${ }^{24} »$. Ainsi, il apparaît clairement qu'il argumente par la théologie, ce type de raisonnement étant à notre sens trop subjectif pour tenir dans une approche purement textualiste.

22 DuponCHEele, p. 68-69.

${ }^{23}$ IBID., p. 68 : «l'idée de l'action instrumentale étant alors à l'évidence celle du feu lui-même dans lequel "chacun sera sale", sans que toutefois l'efficience symbolique propre au sel ne soit métaphoriquement attribuée au feu - ce que font par facilité beaucoup de commentateurs ».

${ }^{24}$ IBID., p. 68-69. 
Elliott opte quant à lui pour la leçon longue, arguant que «the so-called conflate text is characteristic of Mark and should be the deciding factor in the following places as well. [...] And in ix 49

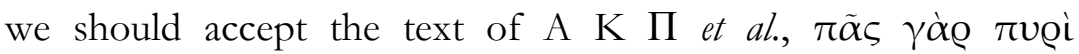

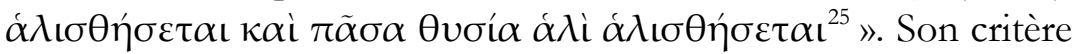
de choix est donc l'accord avec le style de Marc, ce que Focant réfute pourtant en ces termes: "il est peu vraisemblable qu'il faille tenir cette dernière [c'est-à-dire la leçon retenue par Elliott] pour originale au prétexte que Marc aime les "confluences" ou redites ${ }^{26} »$. La leçon longue ayant précédemment été écartée, on sera en outre tenté de retourner cet argument: un copiste a pu combiner les leçons brèves au prétexte qu'il avait remarqué cette tendance littéraire de l'évangéliste.

Reprenons le critère de la lectio difficilior de Duponcheele. C'est

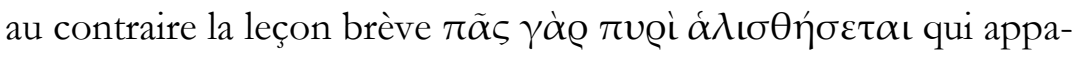
raît comme facilitante, alors que la leçon brève $\pi \tilde{\alpha} \sigma \alpha \gamma \dot{\alpha} \varrho$ Өvoí $\alpha$

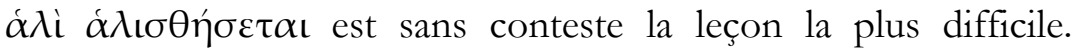
Certes, la leçon retenue par les éditions critiques est « perhaps the most enigmatic logion of Jesus in the $\mathrm{NT}^{27} »$. Pourtant, elle est indubitablement celle qui s'intègre le mieux au contexte dans lequel s'insère le lieu variant. En effet, il est question du feu au v. 48

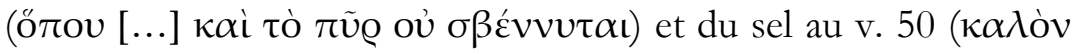

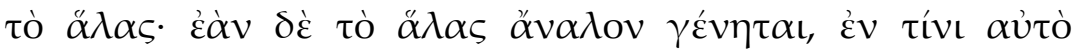
$\alpha \varrho \tau u ́ \sigma \varepsilon \tau \varepsilon ;)^{28}$. Si ces deux notions se retrouvent dans la leçon césaro-alexandrine, seule celle du sel apparaît dans la leçon occiden-

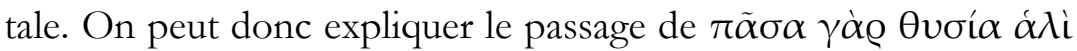

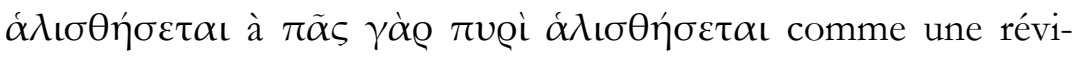
sion visant à rendre le texte plus compréhensible: un copiste a remplacé la mention du sacrifice par celle de l'homme (à travers l'adjectif $\pi \tilde{\alpha} \varsigma$ employé au masculin singulier) et la mention du sel par celle du feu pour lier au mieux la mise en garde et la parole sur le sel dont la consécution n'est pas évidente, ainsi qu'en témoignent les parallèles en Matthieu et en Luc. La leçon brève

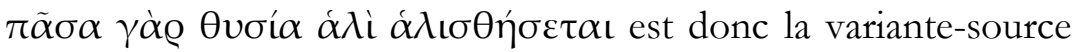

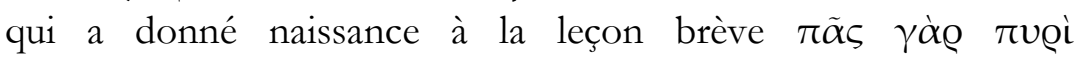

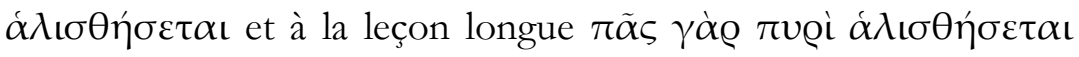

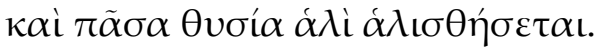

25 J.K. ELLIOTT, The Language and Style of the Gospel of Mark. An Edition of C.H. Turner's 'Notes on Marcan Usage" Together with Other Comparable Studies (Supplements to Novum Testamentum 71), Leiden-New York-Köln, 1993, p. 199.

${ }^{26}$ FOCANT, p. 363.

${ }^{27}$ J. MARCUS, Mark 8-16. A New Translation with Introduction and Commentary (The Anchor Yale Bible 27a), New Haven-London, 2009, p. 698.

${ }^{28}$ LÉGASSE, p. 585 note ainsi très justement que «le "feu" fait le lien avec ce qui précède, le verbe "sera salé" (halisthèsetai) prépare la sentence sur le "sel" (halas) du verset $50 »$. 


\section{Conclusion}

L'examen mené dans cet article a permis assez rapidement d'exclure la leçon longue; les trois étapes de la critique textuelle ont toutes tendu à prouver qu'elle ne peut être que la combinaison des deux leçons brèves. Mais si la critique verbale penchait assez largement en faveur de la leçon $\pi \tilde{\alpha} \varsigma \quad \gamma \dot{\alpha} \varrho \quad \pi v \varrho i$ $\alpha \lambda \iota \sigma \theta \eta \dot{\sigma \varepsilon \tau} \alpha \iota$ - la leçon quasi unanimement retenue comme authentique à ce jour -, la critique externe a commencé à faire pen-

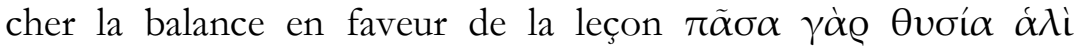
$\alpha \lambda \iota \sigma \theta \eta ́ j \varepsilon \varepsilon \tau \alpha$. La critique interne a quant à elle achevé de nous convaincre de sa primauté, puisqu'elle la valide par deux de ses meilleurs critères : difficilior lectio potior et, surtout, la recherche de la variante-source qui est l'omnium regularum principium de Tischendorf. Par conséquent, on éditera comme texte le plus ancien pour Mc 9,49 celui transmis par les témoins occidentaux parce qu'il présente la leçon qui explique au mieux l'apparition des deux autres. 